\title{
Muography for the Colombian Volcanoes
}

\section{R. de León-Barrios, ${ }^{a}$ J. Peña-Rodríguez, ${ }^{a}$ J.D. Sanabria-Gómez, ${ }^{a}$ A. Vásquez-Ramírez, ${ }^{a}$ R. Calderón-Ardila, ${ }^{b, c}$ C. Sarmiento-Cano, ${ }^{b, c}$ A. Vesga-Ramírez, ${ }^{d}$ D. Sierra-Porta, ${ }^{e}$ M. Suárez-Durán, ${ }^{f}$ H. Asorey ${ }^{b, c}$ and Luis A. Núñez ${ }^{a, g, *}$}

${ }^{a}$ Escuela de Física, Universidad Industrial de Santander, Bucaramanga, Colombia

${ }^{b}$ Instituto en Tecnologías de Detección y Astropartículas, Buenos Aires, Argentina.

${ }^{c}$ Consejo Nacional de Investigaciones Científicas y Técnicas, Buenos Aires, Argentina.

${ }^{d}$ International Center for Earth Sciences, Comisión Nacional de Energía Atómica, Argentina.

e Departamento de Física, Universidad de Los Andes, Bogotá, Colombia.

${ }^{f}$ Université Libre de Bruxelles, Brussels, Belgium.

${ }^{g}$ Departamento de Física, Universidad de Los Andes, Mérida, Venezuela.

E-mail: Inunez@uis.edu.co

We describe the Muography Program to study the Colombian volcanoes. Mainly, we discuss the criteria adopted for designing, building, and commissioning MuTe: a hybrid Muon Telescope. MuTe implements a composite detection technique combining a hodoscope for particle tracking and a water Cherenkov detector for enhancing the muon-to-background-signal separation of extended air showers. Next, we discuss the detailed MuTe digital twin employed to estimate the instrument's response to the muon flux and its impact on its design and performance. The impinging muon flux calculation and its corresponding signals in the detector consider four factors with different spatial and time scales: the geomagnetic effects, the development of extensive air showers in the atmosphere, the propagation through the scanned structure and the detector response at a particular geographic point. Next, we examine the structural -mechanical and thermal- behaviour of $M u T e$, its first calibration measurements and identify the possible volcano candidate with the best observation points. In addition, MuTe incorporates particle-identification techniques for reducing the background noise sources and discrimination of fake events by a picosecond Time-of-Flight system and implementing an offline machine learning framework. We found that the frontal flux adds up $78 \%$ of the recorded events. From this total, $36 \%$ corresponds to electromagnetic component, 33\% single-muon events, and 30\% multiple particle detection. Finally, we discuss an optimization algorithm to improve our instrument's estimation of the volcano's internal density distribution based on the Simulated Annealing method.

$37^{\text {th }}$ International Cosmic Ray Conference (ICRC 2021)

July 12th - 23rd, 2021

Online - Berlin, Germany

\footnotetext{
${ }^{*}$ Presenter
} 


\section{Colombian Volcanoes}

In this report, we review the Colombia Muography Program to study volcanoes, explaining the criteria for designing, building, and commissioning MuTe -the Colombia hybrid Muon Telescope-. We also discuss the first results obtained during the commissioning process.

Any active volcano that erupted in the recent past could erupt again with imminent risks to surrounding populations. Therefore, knowing the internal structure of active volcanoes is crucial for assessing their potential hazards and social consequence. However, geophysical, seismic, gravimetric and geoelectric methods applied to modelling volcano internal composition have spatial limitations and require dense data sampling in severe field conditions [1, 2].

Muography is a non-invasive technique that measures the muon flux attenuation by rock volumes of different densities. The projected images of volcanic conduits help to understand possible eruption dynamics, offering a spatial resolution in the order of tens of metres, up to a kilometre in depth $[2,3]$. Colombia has many volcanoes and more than a dozen active ones

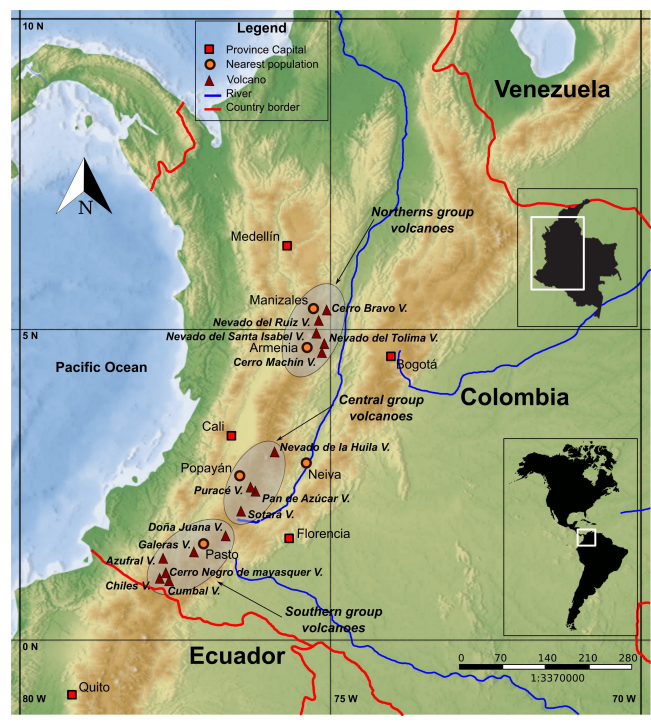

Figure 1: Artistic representation of 13 Colombian active volcanoes spread in three clusters through the Cordillera Central.

represent significant risks to the nearby populations. This has motivated local research groups to apply muography to model inland geological structures. Some preliminary modelling had been reported for devising a muon telescope to study the Galera volcano [5, 6]. C. A. Avila-Bernal and his group designed and built a hodoscope prototype to measure muon flux crossing Monserrate Hill in Bogotá. They determined the muon incident energy's attenuation function and estimated the muon flux as a function of the mountain's location [7].

The most dangerous active Colombian volcanoes are: Azufral, Cerro Negro, Chiles, Cumbal, Doña Juana, Galeras, Machín, Nevado del Huila, Nevado del Ruíz, Nevado Santa Isabel, Nevado del Tolima, Puracé, and Sotará. Figure 1 displays their geographic distribution. 


\section{A muography methodology for Colombian Volcanoes}

There are only a few potential observation points for Colombian volcanoes, commonly surrounded by other geological structures screening the atmospheric muon flux. To identify their possible muon observation sites, we implemented a complete and detailed simulation code considering geomagnetic conditions, atmospheric reaction and detector response [8-10].

In reference [11] we presented the results of ray-tracing analysis for the volcanoes mentioned above. There, we identified Cerro Machín as the best volcano to be studied, spotted the finest points to place a muon telescope and estimated its time exposures for significant statistics of muon flux (see Table 1).

\begin{tabular}{|l|c|c|c|}
\hline Volcanoes & Q 1 & Q 2 & Q 3 \\
\hline Azufral, Cumbal, Dona Juana \& Sotará & N & Y & N \\
Cerro Negro* \& Chiles* & Y & Y & N \\
Galeras & Y & N & Y \\
Machín & Y & Y & Y \\
Nevado del Huila & N & Y & N \\
Nevado del Ruíz, Nevado Santa Isabel \& Puracé & N & Y & Y \\
Nevado del Tolima & N & N & Y \\
\hline
\end{tabular}

Table 1: Which Colombian volcano can be studied by muography? We summarized the answers to the three questions applied to 13 Colombian volcanoes. Q 1- Is the volcano base width less than 1,500 m?. Q 2- The surrounding topography does not interfere with the observation point?. Q 3- Are the sites accessible and secure? Our studies suggested that only Cerro Machín obtains three definite answers. However, we hope that shortly with the internal conflict over, Cerro Negro and Chiles will be safely accessible.

The time exposures of the detector is between 100 to 125 days for the volcano edifice's upper end $(114-150 \mathrm{~m})$ provide $10 \%$ of density discrimination. These results were reconfirmed using more precise muon underground propagation codes $[12,13]$.

\section{The Muon Telescope, MuTe}

MuTe implements a composite detection technique combining a hodoscope for particle tracking and a Water Cherenkov detector (WCD) for enhancing the muon-to-background-signal separation. MuTe employs a plastic scintillator hodoscope to determine the particles' trajectories. The WCD filters the noise due to the soft component of EAS (electrons and positrons) and particles arriving simultaneously. Discrimination of fake events due to scattered and backwards muons is addressed using a picosecond Time-of-Flight system and offline machine learning techniques [14-16].

\subsection{The instrument}

Figure 2 sketches the Colombian hybrid Muon Telescope, MuTe, combining two detection techniques: Two-panel-hodoscope: two X-Y arrays of $30 \times 30$ plastic scintillating strips $(120 \mathrm{~cm} \times 4 \mathrm{~cm} \times 1 \mathrm{~cm})$. Each array has 900 pixels of $4 \mathrm{~cm} \times 4 \mathrm{~cm}=16 \mathrm{~cm}^{2}$, which represent $14,400 \mathrm{~cm}^{2}$ of panel detection surface. These panels can be separated up to $D=250 \mathrm{~cm}$; Water 


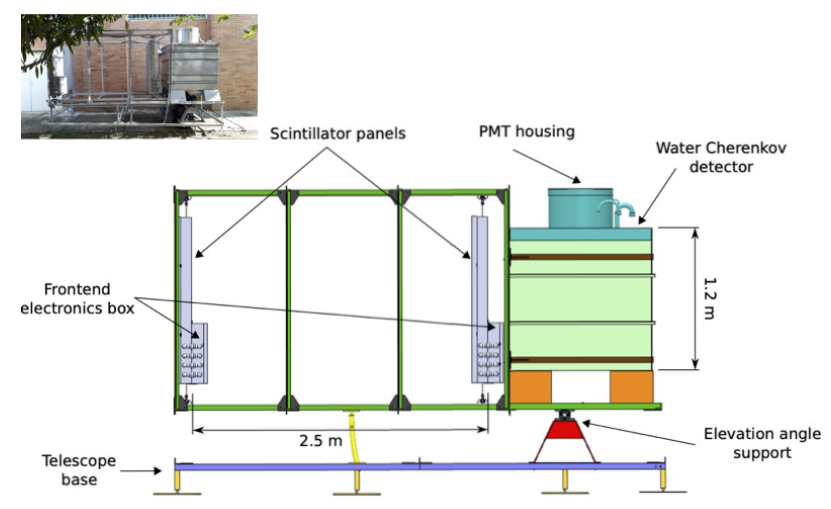

Figure 2: A sketch of the Colombian Muon Telescope (MuTe), which combines the facilities of a two-panelhodoscope (900 pixels) and a $1.73 \mathrm{~m}^{3}$ water Cherenkov detector.

Cherenkov Detector: a purified water cube of $120 \mathrm{~cm}$ side, located behind the rear scintillator panel (see figure 2), which acts as a calorimeter and as a third active coincidence detector.

Designing the structure's reaction (vibration frequencies not exceeding $0.04 \mathrm{~Hz}$ ) guarantees its structural integrity against seismic events triggered by volcanic activity [15]. Furthermore, under a maximum wind speed of $30 \mathrm{~m} / \mathrm{s}$ (occurring in $\approx 54$ days/year), the mechanical structure suffers displacements of up to $1.63 \mathrm{~mm}$ in the frontal part of the scintillator panel. Thus, deformations under stresses due to wind are negligible. Finally, we also performed the thermal structure analysis. The WCD is a good heat dissipator due to the water content $\left(\sim 1.7 \mathrm{~m}^{3}\right)$ attaining a maximum temperature of $40^{\circ} \mathrm{C}$. In this case, the front side of both panels has a lower temperature than the rear side since the wind flow generates a cooling process by convection.

\subsection{MuTe digital twin}

Reference [17] compared the response of the MuTe Geant4 digital twin [18]. From the scintillator simulation, we obtain $7 \%$ reductions in the number of photo-electrons, compared to those produced at the near end of the silicon photo-multiplyers, SiPMs. The more distance the photo-electrons travel within the fibre, the more energy they lose, and fewer photons reach the SiPMs. This result is in agreement with the experimental 9\% attenuation obtained. This attenuation seems to be insignificant in the bar. Still, it is more noticeable in the hodoscope panels since the difference between the closest corner to the SiPMs and the furthest is around $12 \%$.

MuTe Geant4 simulations estimate the detection trigger in terms of the energy deposited by each component. First, muons must deposit about $2.08 \mathrm{MeV}$ in the two scintillator bars on the front panel and finally discriminated from the background in the WCD where muons must lose about $240 \mathrm{MeV}$ to be counted as an event.

\subsection{The intrumentation}

The MuTe electronics has two leading -independent but synchronized-readout systems: one for the hodoscope and one for the WCD. A multi-channel ASIC MAROC3 (from Omega) discriminates the 60 signals after making a gain adjustment to reduce the bar response variability. An FPGA 
Cyclone III sets the MAROC3 slow control parametres (channel gains and discrimination thresholds) from Altera [19].

A local server collects data from the WCD and the hodoscope every 12 hours for carrying out an in-situ analysis. An intranet system connects the local server, the hodoscope, and the WCD. Additionally, the MuTe enables a wireless access point for working locally from a laptop or any other mobile device.

The five-level triggering system determines event coincidence between the hodoscope and the WCD. Time delays due to the transmission of the signals are estimated and considered for data analysis.
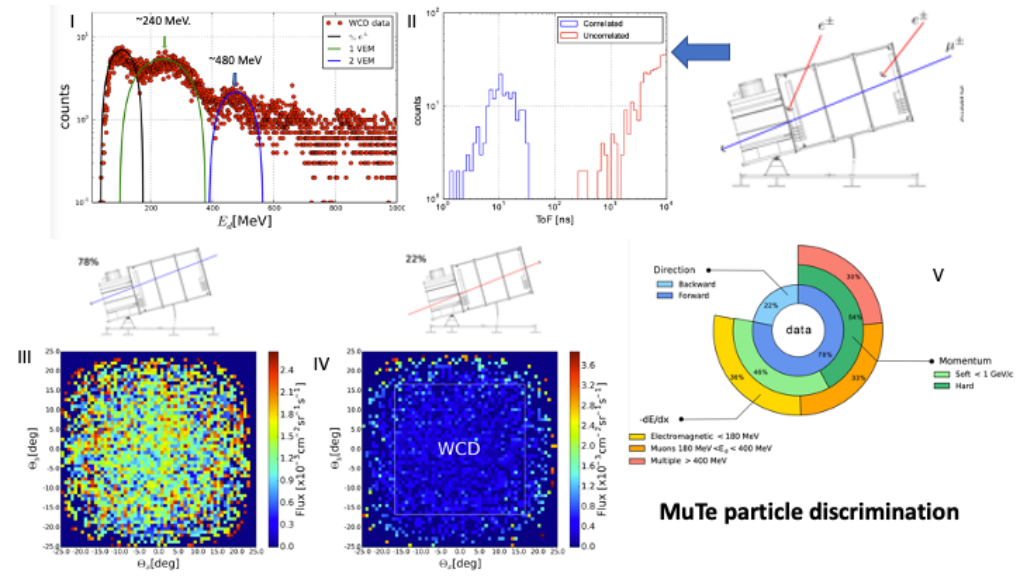

Figure 3: The upper Left, plate I plots the deposited energy histogram for the average number of events crossing the MuTe during one hour. The black-line hump $(<180 \mathrm{MeV})$ matches the electromagnetic component $(e \pm \gamma)$, while the green-line represents the deposited energy of muons: $180 \mathrm{MeV}<E_{d}<400 \mathrm{MeV}$. The blue-line bump expresses two-muon events. Multiple particle events deposit energy above $400 \mathrm{MeV}$. Next, the upper right, plate II displays the Time-of-Flight of particles traversing the hodoscope. Singleparticle events and correlated background (blue) have a ToF $<30$ ns. The uncorrelated ones (red) has a $\mathrm{ToF}>300 \mathrm{~ns}$. At the bottom, plates III and IV illustrate, respectively, the angular distribution of the frontal and the inverse particle flux traversing the hodoscope with an elevation angle of $15^{\circ}$ and the axis located at $\Theta_{x}=\Theta_{y}=0 \mathrm{deg}$. The frontal flux adds up $78 \%$ of the recorded events reaching up to $3.23 \mathrm{~Hz}$. The WCD volume (white-square) absorbs the particles with energy $<240 \mathrm{MeV}$. Plate $V$ displays the incoming particle distribution: 36\% electromagnetic component, 33\% single-muon events, and 30\% multiple particle detection. See reference [16] for details.

MuTe power supply has four photovoltaic panel array of $100 \mathrm{~W}(18 \mathrm{~V}-5.56 \mathrm{~A})$, which provides the instrument with six days of continuous operation, the maximum number of consecutive cloudy days in the last 22 years at the observation site.

\subsection{MuTe particle discrimination and first muogram}

Figure 3 (plate I) shows the average hourly count of events recorded in WCD-Hodoscope coincidence and can be divided into three categories. The electromagnetic component (photons, electrons and positrons) with an energy loss of less than $180 \mathrm{MeV}$ represents $36 \%$ of the events. The muon part makes up $33.6 \%$ of the events in the range $180 \mathrm{MeV}<E_{d}<400 \mathrm{MeV}$ with an 
average of $240 \mathrm{MeV}$. Finally, multiple particle events dissipate above $400 \mathrm{MeV}$ with a significant contribution at $480 \mathrm{MeV}$, adding up the $\approx 30 \%$ of the effectively recorded impacts [16].

We confirmed the WCD particle identification by implementing several machine learning algorithms. We apply a supervised Naive Gaussian, Support Vector Machines (Radial Basis Function), and Random Forest. The Naive Gaussian classified the components with higher precision: $99 \%$ of the electromagnetic particles, $100 \%$ of muons, and $97 \%$ multiple particle events.

As shown in plate II 3, the precise time of flight, ToF, implemented for MuTe, determined that single-particle events span from $2.53 \mathrm{~ns}$ to $20.9 \mathrm{~ns}$, and the uncorrelated background starts at $\approx 300 \mathrm{~ns}$. Therefore, the probability that an uncorrelated event occurs below $\approx 200 \mathrm{~ns}$ is rough $0.05 \%$.

In plates III and IV of figure 3, we compare the frontal (left) and the inverse (right) flux crossing. The inverse events represent $22 \%$ of particles impinging the detector, and the WCD absorbs most of these particles with energy $<240 \mathrm{MeV}$ (see reference [16] for details).

Form two-month of recorded data, we obtain the first muogram shown in figure 4 . With $15^{\circ}$ elevation, the telescope aims at a mountain at $\approx 20 \mathrm{~km}$ from the detector, having a maximum altitude of 3466 m.a.s.1.

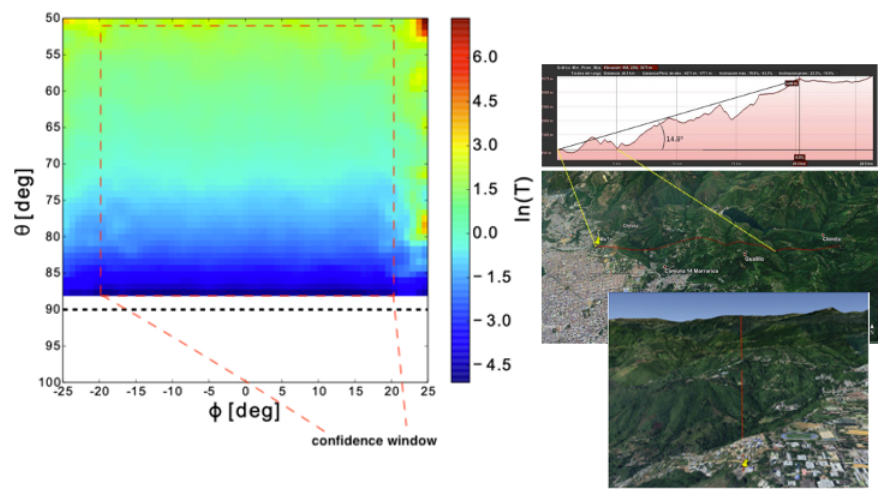

Figure 4: Left plate displays the muogram which is the logarithm of the transmittance, i.e. $T=\ln \left(\frac{\Phi}{\Phi_{0}}\right)$ with $\Phi$ the particle flux and $\Phi_{0}$ open sky particle flux. For the open sky areas $\left(\theta>70^{\circ}\right)$ the transmittance is $\approx 1 \approx \ln (T)=0$ ). Below the horizontal (dotted line), the open sky flux is zero. MuTe's two months of recorded flux at an elevation of $15^{\circ}$. On the right composition we illustrate the detector line of sight (red line). The instrument's elevation $\approx 15^{\circ}$ covered a zenith range of $50^{\circ}$ to $90^{\circ}$ and $10^{\circ}$ below the horizon. Notice the confidence window negleting measuremente from large angles.

\section{Optimization of density distribution}

In [21] we describe an optimization method for obtaining the best density distribution inside the volcano, instead of using a Bayesian framework [22-24] or local optimization methods [1, 25], we study the geophysical inversion problem with the Simulated Annealing Algorithm (SAA) applying the Metropolis criterion [26]- to obtain the global optimum for the density distribution function inside the volcano. It is computationally efficient, can solve non-linear problems, and does not introduce smoothing effects in the final density structure model [27]. 
The estimated initial density model of the dome was obtained with GEOMODELER, adapted to the Cerro Machín topography. We have improved this model by including the rock densities of samples taken from the crater, the dome and the areas associated with fumaroles.

The inversion algorithm correctly reconstructed the density differences inside the Machín, within a $1 \%$ error concerning our initial simulation model, giving a remarkable density contrast between the volcanic duct, the encasing rock and the fumaroles area.

\section{Acknowledgments}

The authors acknowledge the financial support of Departamento Administrativo de Ciencia, Tecnología e Innovación of Colombia (ColCiencias) under contract FP44842-082-2015. We thank LAGO and the Pierre Auger Collaboration for their continuous support and inspiration..

\section{References}

[1] M. Rosas-Carbajal et al. Three-dimensional density structure of la soufrière de guadeloupe lava dome from simultaneous muon radiographies and gravity data. Geophys. Res. Lett., 44(13):6743, 2017.

[2] H.K.M. Tanaka et al. Imaging the conduit size of the dome with cosmic-ray muons: The structure beneath showa-shinzan lava dome, japan. Geophys. Res. Lett., 34(22):L22311, 2007.

[3] N. Lesparre et al. Geophysical muon imaging: feasibility and limits. Geophys. J. Int., 183(3):1348, 2010.

[4] S. Okubo et al. Imaging the density profile of a volcano interior with cosmic-ray muon radiography combined with classical gravimetry. Meas. Sci. Technol., 23(4):042001, 2012.

[5] A. Tapia et al. First monte carlo simulation study of galeras volcano structure using muon tomography. PoS, ICHEP2016:885, 2016.

[6] I.D. Guerrero et al. Design and construction of a muon detector prototype for study the galeras volcano internal structure. In J. Phys. Conf. Ser., 1247: 012020, 2019.

[7] J.S. Useche-Parra et al. Estimation of cosmic-muon flux attenuation by monserrate hill in bogota. J. Instrum., 14(02):P02015, 2019.

[8] H.Asorey et al. The LAGO space weather program: Directional geomagnetic effects, background fluence calculations and multi-spectral data analysis. PoS, ICRC2015:142, 2015.

[9] H. Asorey et al. Preliminary results from the latin american giant observatory space weather simulation chain. Space Weather, 16(5):461, 2018.

[10] C. Sarmiento-Cano et al. Performance of the lago water cherenkov detectors to cosmic ray flux. arXiv:2010.14591, 2020.

[11] A. Vesga-Ramírez et al. Muon tomography sites for colombian volcanoes. Ann. Geophys., 63(6):661, 2020. 
[12] V.A. Kudryavtsev. Muon simulation codes music and musun for underground physics. Comput. Phys. Commun., 180(3):339, 2009.

[13] H. Moss et al. Muon tomography for the cerro machín volcano. Technical report, Dept. of Physics \& Astronomy, Univ. of Sheffield, Sheffield, UK, 2018.

[14] H. Asorey et al. Astroparticle projects at the eastern colombia region: facilities and instrumentation. Scientia et technica, 23(3):391, 2018.

[15] J. Peña-Rodríguez et al. Design and construction of MuTe: a hybrid muon telescope to study colombian volcanoes. J. Instrum., 15(09):P09006, 2020.

[16] J. Peña-Rodríguez et al. Characterization of the muography background using the muon telescope (mute). PoS, ICHEP2020:984, 2021.

[17] A. Vásquez-Ramírez et al. Simulated response of mute, a hybrid muon telescope. J. Instrum., 15(08):P08004, 2020.

[18] S. Agostinelli et al. Geant4: a simulation toolkit. Nucl. Instrum. Methods. Phys. Res. B, 506(3):250, 2003.

[19] J. Sánchez-Villafrades. Diseño e implementación de un sistema de caracterización para los SiPM del telescopio de muones MuTe. Bachelor thesis, Univ. Industrial de Santander, 2020.

[20] J. Peña-Rodríguez et al. Calibration and first measurements of mute: a hybrid muon telescope for geological structures. PoS, ICRC2019:36, 2019.

[21] A. Vesga-Ramírez et al. Simulated annealing for volcano muography. J. South Am. Earth Sci., 109:103248, 2021.

[22] A. Barnoud et al. Bayesian joint muographic and gravimetric inversion applied to volcanoes. Geophys. J. Int., 218(3):2179, 2019.

[23] R. Nishiyama et al. 3d density modeling with gravity and muon-radiographic observations in showa-shinzan lava dome, usu, japan. Pure Appl. Geophys., 174(3):1061, 2017.

[24] R. Nishiyama et al. Integrated processing of muon radiography and gravity anomaly data toward the realization of high-resolution 3-d density structural analysis of volcanoes: Case study of showa-shinzan lava dome, usu, japan. J. Geophys. Res. Solid Earth, 119(1):699, 2014.

[25] P.G. Lelièvre et al. Joint inversion methods with relative density offset correction for muon tomography and gravity data, with application to volcano imaging. Geophys. J. Int., 218(3):1685, 2019.

[26] S. Kirkpatrick et al. Optimization by simulated annealing. Science, 220(4598):671, 1983.

[27] S. Nagihara et al. Three-dimensional gravity inversion using simulated annealing: Constraints on the diapiric roots of allochthonous salt structures. Geophysics, 66(5):1438, 2001. 